\title{
APLICATIVO MÓVEL PARA PREVENÇÃO DE ERROS DE MEDICAÇÁO: PREVMED
}

\author{
MOBILE APPLICATION FOR ERROR PREVENTION \\ IN MEDICATION: PREVMED
}

\section{APLICACIÓN MÓVIL PARA LA PREVENCIÓN DE ERRORES EN LA MEDICACIÓN: PREVMED}

\author{
Marciele Misiak Caldas* \\ Francis Solange Vieira Tourinho** \\ Vera RadünZ*** \\ Vivian Costa Fermo**** \\ Patricia IllaA ${ }^{* * * * *}$ \\ Thaís Fávero Alves******
}

\begin{abstract}
RESUMO
O objetivo deste artigo é descrever o processo de desenvolvimento de um protótipo de aplicativo para dispositivo móvel, para treinamento da equipe de saúde em relaçáo ao uso de um protocolo de prevenção de erros de medicação potencialmente perigosa. A metodologia foi baseada no modelo conceitual de Garret e o desenvolvimento das telas foi por meio do software de prototipação Adobe Experience Design ${ }^{\oplus}$. O protótipo possui uma tela inicial com o nome PrevMed, seguida por uma tela de menu que compóe um link de acesso à lista de Medicamentos Potencialmente Perigosos (MPPs), cinco domínios, um ícone para treinamento e um ícone de referências, com um total de 30 telas. Aqui serão apresentadas 13 telas que exemplificam cada uma das açôes possíveis no aplicativo. O protótipo do aplicativo fornecerá ao profissional o acesso à informação com facilidade e rapidez, contribuindo a seu treinamento tanto para o conhecimento de quais são os MPPs, assim como as açóes necessárias para continuar com o processo.
\end{abstract}

Palavras-chave: Protocolos; Aplicativos Móveis; Segurança do Paciente; Erros de Medicação; Tecnologia.

*Enfermeira. Doutora em Enfermagem, Instituto Federal de Santa Catarina, Florianópolis, Brasil. Email: marciele.misiak@gmail. com. Autor correspondente.

**Enfermeira. Doutora em Saúde da Criança e do Adolescente. Departamento de Enfermagem, Universidade Federal de Santa Catarina, Florianópolis, Brasil. Email: francis.tourinho@ufsc.br

***Enfermeira. Doutora em Enfermagem. Departamento de Enfermagem, Universidade Federal de Santa Catarina, Florianópolis, Brasil. Email: vera.radunz@ufsc.br

****Enfermeira. Doutoranda em Enfermagem, Prefeitura Municipal de Florianópolis, Florianópolis, Brasil. Email: vivianfermo@ hotmail.com

*****Enfermeira. Doutora em Enfermagem. Departamento de Enfermagem Universidade Federal de Santa Catarina, Florianópolis, Brasil. Email: ilha.patricia@gmail.com

******Enfermeira. Doutoranda em Enfermagem, Universidade Federal de Santa Catarina, Florianópolis, Brasil. Email: thais. faveroalves@gmail.com 


\section{ABSTRACT}

The purpose of this article is to report on the process of developing a mobile device application prototype for training the healthcare staff regarding the use of a protocol for the prevention of errors with potentially dangerous drugs. The methodology is based on Garrett's conceptual model and the development of screens using the Adobe Experience Design prototyping software. The prototype has a splash screen with the name PrevMed, followed by a menu screen that includes a link to the list of Potentially Dangerous Drugs (PDD), the five domains, a training icon and a reference icon, totalizing 30 screens. 13 different screens will display each of the possible actions in the application. The application prototype will provide the health professional quick and easy access to the information, also contributing to their qualification both for the knowledge of the PDDs and the necessary actions to be followed during the process.

Keywords: Protocols; Mobile Applications; Patient Safety; Medication Errors; Technology.

\section{RESUMEN}

El propósito de este artículo es describir el proceso de desarrollo de un prototipo de una aplicación para dispositivo móvil, cuyo objetivo es capacitar al equipo de salud respecto al uso de un protocolo de prevención de errores en medicamentos potencialmente peligrosos. La metodología se basó en el modelo conceptual de Garret y el desarrollo de las pantallas a través de software de prototipado Adobe Experience Design ${ }^{\oplus}$. El prototipo tiene una pantalla inicial con el nombre PrevMed, seguida de una pantalla menú que incorpora un enlace de acceso a la lista de Medicamentos Potencialmente Peligrosos (MPPs), cinco dominios, un ícono para capacitación y un ícono de referencias, totalizando 30 pantallas. Aquí se presentan 13 pantallas que ejemplifican cada una de las acciones posibles en la aplicación. El prototipo de la aplicación proporcionará al profesional acceso a información de forma fácil y rápida, contribuyendo a su capacitación tanto para el conocimiento de cuáles son los MPPs, como las acciones necesarias a seguir durante el proceso.

Palabras clave: Protocolos; Aplicaciones Móviles; Seguridad del Paciente; Errores de Medicación; Tecnología.

Fecha de recepción: 19/08/2019

Fecha de aceptación: 10/12/2019

\section{ERROS DE MEDICAÇÃO E OS MEDICAMENTOS POTENCIALMENTE PERIGOSOS}

Apesar dos esforços mundiais para a segurança do paciente, com iniciativas que permeiam metas e programas de melhorias em instituiçóes de saúde, o número de eventos adversos que ocorrem no cotidiano hospitalar ainda é expressivo. Estudo realizado em um hospital de ensino brasileiro ${ }^{(1)}$, por exemplo, identificou a ocorrência de 324 Eventos Adversos (EA) em 115 pacientes. A taxa de incidência foi de 9,3 EA por 100 pacientes-dia, o que gerou um aumento do tempo de internação e da mortalidade. Os EA têm um impacto expressivo aos pacientes e as instituiçóes de saúde, assim, é necessário que as instituiçóes conheçam seus indicadores, para que possam planejar açóes e promover cuidado seguro.

Dentre os EA, aqueles relacionados aos erros de medicação estão entre os mais comuns. Em uma Unidade de Terapia Intensiva no Chile ocorreram 625 incidentes com uma média de 0,7 incidentes por paciente, sendo que os erros de medicação representaram $89,56 \%{ }^{(2)}$. No Brasil, estudo que analisou os EA ocorridos em uma clínica médica apontou que dos 1.360 eventos ocorridos, 21,2\% foram erros de medicação ${ }^{(3)}$.

Dentre os erros com medicamentos, os relacionados aos medicamentos potencialmente perigosos (MPP), apesar de não serem os maios comuns, acarretam em consequências mais devastadoras para os pacientes ${ }^{(4)}$. Um estudo analisou a prática da prescriçáo de medicamentos 
potencialmente perigosos e sua relação com a prevalência de erros de medicaçáo em ambiente hospitalar. Foram avaliadas 4.026 prescrições, nessas prescriçóes foram encontrados 7.148 MPP e, nestes 7.148 MPP foram detectados 3.177 (44,5\%) $\operatorname{erros}^{(5)}$.

O Instituto para Práticas Seguras no uso dos Medicamentos (ISMP) elaborou listas elencando os MPP para uso hospitalar, ambulatorial e para instituiçóes de longa permanência, e apresentou algumas recomendaçóes para a prevenção de erros. Dentre as recomendaçóes está a elaboração de protocolos ${ }^{(4)}$. O protocolo é uma tecnologia utilizada com o intuito de padronizar açóes, é uma ferramenta importante na área da saúde e tornou-se essencial para a gestão da assistência e a promoção da segurança do paciente ${ }^{(6)}$.

A utilização dos protocolos modifica o comportamento da equipe de saúde quando utiliza das estratégias adequadas para sua implementação e sugerem-se alternativas interativas para que o profissional se torne mais ativo no processo e não somente a disseminação de materiais impressos ${ }^{(7)}$. Uma das formas sugeridas para promoção dessa interação do profissional com diversos conteúdos e, consequentemente, pode ser empregue para adesão aos protocolos, é a utilização de tecnologias como os aplicativos para dispositivos móveis.

O uso de aplicativos favorece agilidade na busca de informaçóes, beneficia a precisão do cuidado devido a açóes como apoio à tomada de decisão, ao diagnóstico e a possibilidade de monitoramento remoto ${ }^{(8)}$. Acredita-se que, para construir um aplicativo que contemple o conteúdo de um protocolo, ele deve apresentar as açóes que devem ser realizadas em cada etapa do uso do MPP, para fornecer barreiras que evitem erros, que contribuam para o treinamento da equipe e melhorem a segurança do paciente.

Sabe-se que na área da saúde há resistência e restrições das instituiçóes e dos próprios órgãos que regulamentam as profissóes quanto ao uso do smartphone dentro das unidades de atendimento ao paciente. Estudo realizado na Austrália analisou como é o uso da tecnologia pela enfermagem e avaliou que seu uso é restrito, há atenção excessiva pelo medo do não cumprimento de regras e normas das instituiçôes, o que reflete na dificuldade de acesso a diversas informaçóes. São necessárias discussóes sobre o tema de forma a atender um ponto de equilíbrio, visto que a profissão vem sendo prejudicada pela falta de acesso à informação proporcionada pelo uso de celulares no momento da assistência ao paciente ${ }^{(9)}$.

O advento do uso da tecnologia é irreversível e a sua utilização tem registrado benefícios ao atendimento ao paciente de modo a colaborar para um cuidado seguro. Os smartphones possuem o benefício de ser transportado com facilidade, o que otimiza o seu uso a beira do leito ${ }^{(10)}$. A utilização adequada da tecnologia favorece os profissionais, apresenta benefícios como a otimização do tempo, a promoção da qualidade do cuidado e contribui para a capacitação e melhora da competência dos profissionais ${ }^{(11)}$. Portanto conscientizar a equipe do uso correto dos smartphones é um desafio dentro das instituições de saúde para que sejam utilizados em benefício dos profissionais e pacientes.

Estudo que elaborou um protótipo de um aplicativo para registro de dados da sistematização da assistência de enfermagem (SAE), em unidade de terapia neonatal, mostrou que houve uma necessidade de adaptação ao seu uso e a superação de obstáculos, principalmente relacionados ao manuseio do tablet, porém, os benefícios foram superiores e destaca-se a flexibilização das atividades do enfermeiro, além da aderência às etapas da SAE que é uma das grandes resistências da enfermagem ${ }^{(12)}$.

Identifica-se que o uso de aplicativos na capacitação dos profissionais durante o seu cotidiano pode favorecer a busca acessível de informaçóes atualizadas e minimizam o risco da realização de uma ação incerta no momento da assistência ao paciente.

Diante do exposto, esse artigo tem como objetivo relatar o processo de desenvolvimento de um protótipo de aplicativo para dispositivo móvel, para capacitação da equipe de saúde referente ao uso de um protocolo de prevenção de erros de medicamentos potencialmente perigosos.

\section{DESENVOLVIMENTO DO PROTÓTIPO}

Para o desenvolvimento do protótipo foi utilizado o modelo conceitual de Garret que utiliza a metodologia centrada na experiência do usuário, a web como software, visando à validação antes de sua implementação. Esse modelo é divido em 
algumas etapas para que se parta do abstrato e chegue ao concreto: necessidades do usuário objetivo do site; especificaçóes funcionais, design de interação/arquitetura da informação; design da informação e design visual ${ }^{(13)}$.

O protótipo foi desenvolvido em parceria com um profissional do Design, sendo que, para chegar ao seu desenvolvimento, foi necessária a realização de duas etapas anteriores que antecederam a esta construção aqui relatada, sendo elas: a elaboração de protocolo para a prevenção de erros com MPP; e a validação do protocolo de prevenção de erros com MPP.

Foram realizados cinco encontros entre pesquisadora e a profissional do design durante os meses de agosto a outubro de 2017. Nos encontros foram apresentadas, pelo pesquisador ao profissional do Design, as necessidades do usuário, que se transformaram nos domínios validados no protocolo (descritos a seguir) e o objetivo do aplicativo. Assim, foi definido que seriam utilizadas na prototipação do aplicativo as informaçóes contidas no protocolo para a prevenção de erros com MPP.

Foi disponibilizado o conteúdo do protocolo ao profissional do Design para dar seguimento a prototipação da tecnologia. Em seguida verificouse a pertinência de disponibilizar acesso rápido a lista de MPP e aos seis domínios definidos no protocolo validado e que serâo apresentados nos resultados deste estudo.

Assim, no primeiro e segundo encontro discutiuse sobre os itens que comporiam o aplicativo e no segundo encontro decidiu-se que do sexto domínio do protocolo de prevençáo de erros com MPP, seria utilizado somente o item Capacitação, visto que os demais itens (organização do ambiente físico, envolvimento do enfermeiro e farmacêutico, monitorar indicadores, número de recursos humanos e jornada de trabalho, briefing e debrief) alguns foram considerados eixos transversais $\mathrm{e}$ outros não estariam de acordo com o escopo do aplicativo no momento.

Com essas informaçóes foram desenvolvidas algumas telas e identificou-se o check list como a melhor forma para qualificar a interação do usuário com a ferramenta. Posteriormente foram elaboradas as telas com os conteúdos completos procurando sempre apresentar funcionalidade e facilidade de acesso ao usuário. $\mathrm{O}$ desenvolvimento das telas se deu por meio de um software de prototipação Adobe Experience Design ${ }^{\oplus}$, que permite ao profissional do design uma prototipagem rápida, fluida e que possibilita o desenvolvimento de apps e sites de forma prática e eficiente.

No terceiro encontro foram apresentadas as telas iniciais, que foram discutidas e aperfeiçoadas durante o quarto e quinto encontro. Atualmente o protótipo está pronto para futura validação com os usuários.

Cabe ressaltar que este trabalho foi desenvolvido somente após aprovação do mesmo pelo Comitê de Ética em Pesquisa (CEP) sob o parecer de número 1.642 .831 .

\section{APRESENTAÇÃO DAS TELAS}

O protótipo é composto por uma tela inicial, seguida de uma tela Menu que compóe um link de acesso a lista de MPP contendo os cinco domínios do protocolo de prevenção e erros com MPP (Prescrição médica; Armazenamento, separação e distribuições dos medicamentos; Preparo dos medicamentos; Administração dos medicamentos; Cuidados pós administração), e um ícone para capacitação (proveniente do sexto domínio do protocolo), além de outro ícone de referências, totalizando 29 telas.

A seguir serão apresentadas 13 telas que exemplificam cada uma das atividades possíveis no aplicativo, como também serão descritas quais as possibilidades em cada etapa que totalizam as 29 telas.

A Figura 1 apresenta as quatro telas iniciais do aplicativo. A primeira tela contém o nome representativo PrevMed-Prevenção de Erros com Medicamentos Potencialmente Perigosos. A segunda, o menu que permite o acesso a lista de MPPs de uso hospitalar e o acesso aos cinco primeiros domínios do protocolo, que são as etapas de administração destes medicamentos já descritas anteriormente. E por fim o acesso à sessão de capacitação (representa o sexto domínio) e às referências. A terceira e a quarta tela apresentam a busca conforme a lista dos MPP de uso hospitalar do Instituto para Práticas Seguras no uso de Medicamentos $^{(4)}$, sendo a terceira os medicamentos por classe terapêutica e a quarta por medicamento específico. 

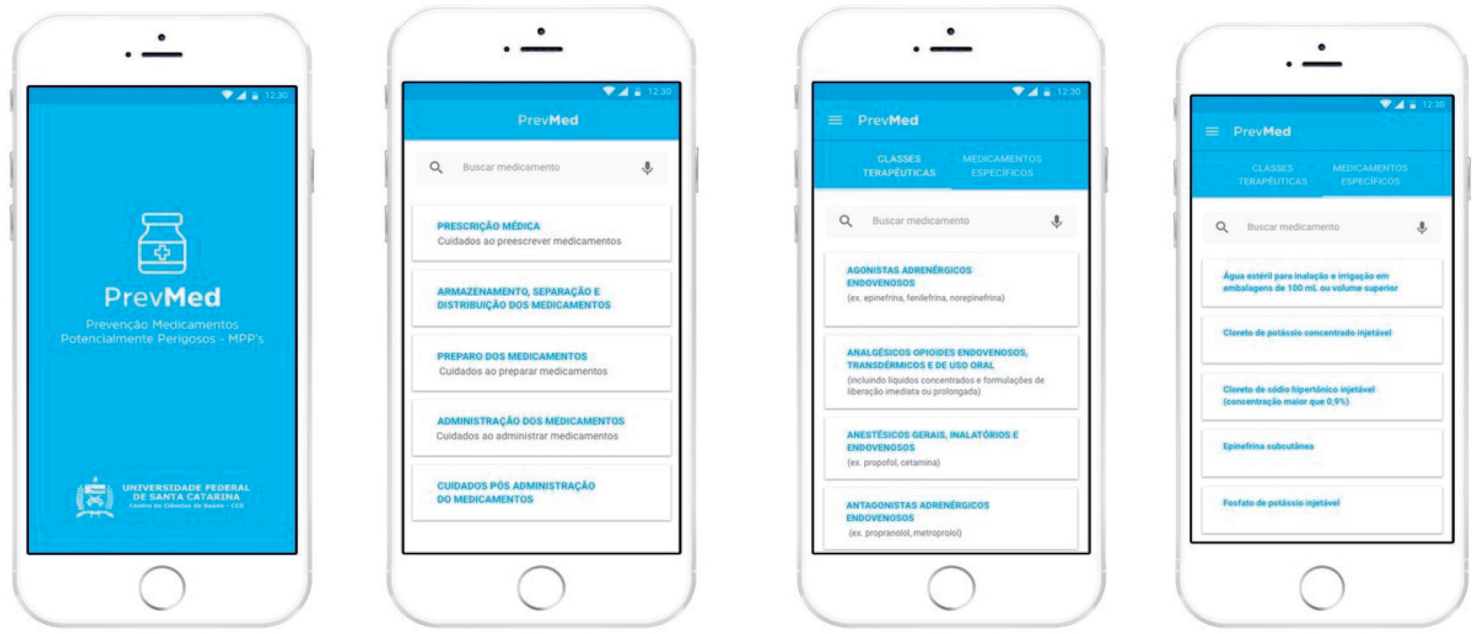

Figura 1: Tela inicial; Menu: busca, acesso aos domínios e capacitação; Lista MPP: Busca por classe terapêutica; Lista MPP: Busca por medicamento específico.

A Figura 2 apresenta na primeira tela, "Prescrição", os cuidados a serem realizados pelo médico no momento da prescrição, que são divididos em sete itens: legibilidade, identificação, padronização, identificação visual, alertas, certos da prescrição e farmacêutico clínico. Na segunda tela, "Armazenamento, separação e distribuição", se encontram os cuidados referentes à farmácia, divididos em 10 itens: ambiente, infraestrutura, armazenamento, etiquetas de identificação, análise da prescrição, separação, conferência, código de barras, dupla checagem e sistema de dispensação. $\mathrm{Na}$ terceira tela, "Preparo", são elencados os cuidados utilizados no momento do preparo dos medicamentos, divididos em seis itens: acesso à lista MPP, tipo de dispensação, local do preparo, zona de não interrupção/colete, certos do preparo, açóes durante o preparo. Na primeira, segunda e terceira tela, presentes na imagem, é possível acessar outras telas referentes às orientações específicas ao clicar no ícone "?". A quarta tela, denominada "Orientaçóes específicas dos itens”, exemplifica os "?" que estão ao lado de alguns itens em todos os domínios e dá acesso a informações mais detalhas e justificativas daquele item.
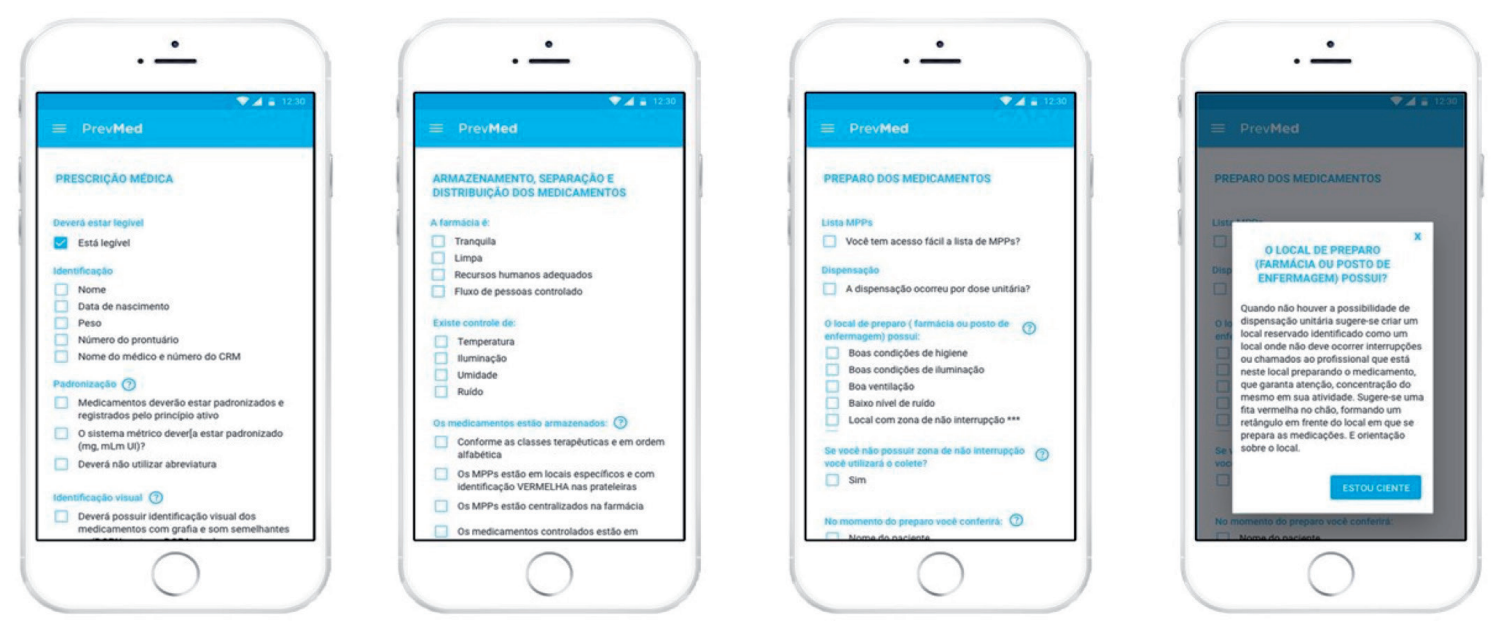

Figura 2: Prescrição; Armazenamento, separação e distribuição; Preparo; Orientações específicas dos itens. 
A Figura 3 segue apresentando as etapas do processo de uso de medicamentos, sendo a primeira tela referente a "Administração" e apresenta o momento de administração dos medicamentos. Nessa etapa foram elencados quatro itens: certos da administração, cuidados antes de administrar, cuidados no momento da administração e logo que administrar. A segunda tela, "Cuidados pós-administração", tem um item que orienta o que deverá ser realizado após a administração do medicamento. Na primeira e segunda tela, presente na imagem, é possível acessar outras quatro telas referentes às orientaçóes específicas. A terceira tela, "Feedback", trata-se de um feedback para os casos em que caso o profissional não assinalar algum dos itens em qualquer dos domínios aparecerá uma tela questionando se ele tem certeza e informando que ao deixar um dos itens sem execução, ele estará correndo risco em relação a segurança do uso dos medicamentos potencialmente perigosos, e para que ele saia dessa tela ele marcará que está ciente. A quarta tela, "Capacitação", apresenta uma tela cujo conteúdo será desenvolvido futuramente. Este local será destinado a capacitação dos profissionais em relação aos MPPs. Neste serão inseridos links de artigos sobre o tema, criadas atividades interativas e lúdicas para capacitar a equipe de saúde. A quinta tela, "Referências", apresenta as referências utilizadas para a construção do protocolo que embasou o conteúdo do aplicativo.
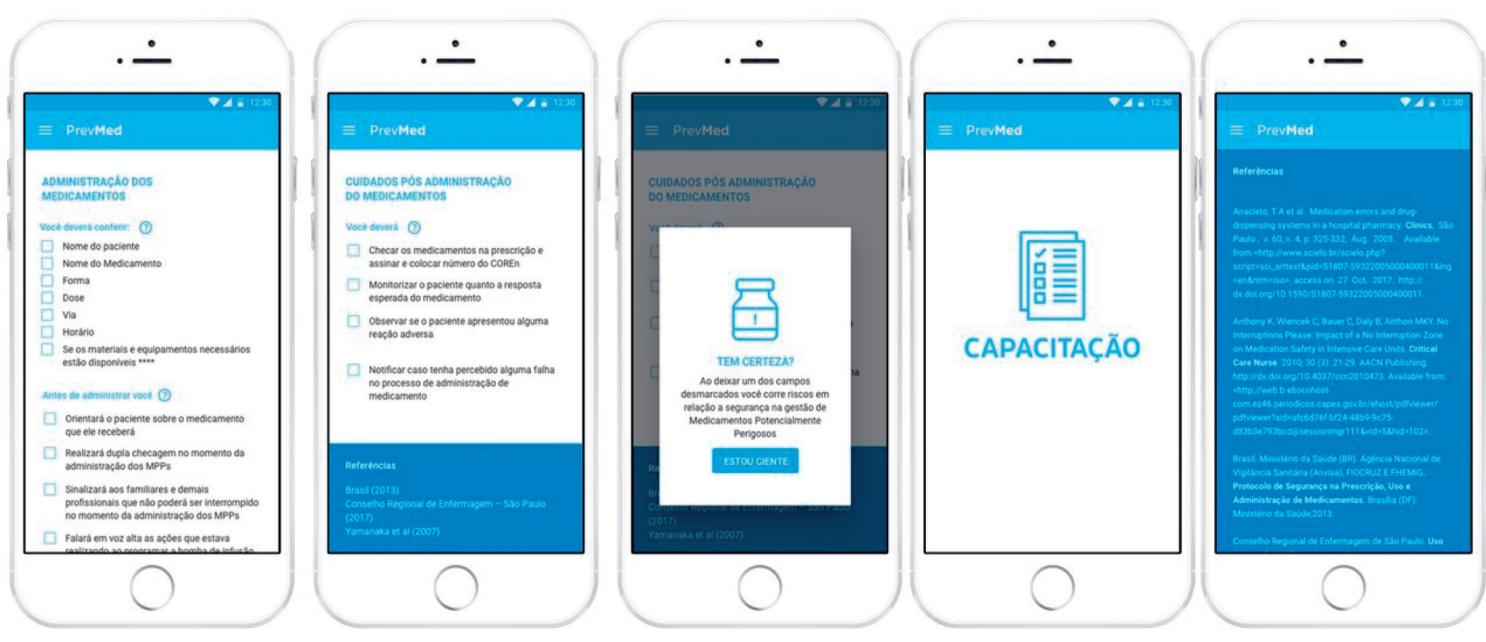

Figura 3: Administração; Cuidados pós administração; Feedback; Capacitação; Referências.

\section{IMPLICAÇÓES À PRÁTICA PROFISSIONAL}

O grande desafio no desenvolvimento do protótipo do aplicativo para prevenção de erros com MPP foi sintetizar os cuidados de cada etapa do processo do uso de medicamentos, de forma a torná-lo prático, completo e exequível.

No protótipo do aplicativo desenvolvido nesse estudo a tela do Menu apresenta a possibilidade de o profissional acessar a lista de MPP de uso hospitalar. Uma das orientaçóes do Instituto para Práticas Seguras no Uso de Medicamentos - ISMP é o contato fácil a essa lista ${ }^{(4)}$. A lista no aplicativo será atualizada conforme atualização realizada pelo ISMP.
Posteriormente no PrevMed será incluído um link para as bulas, o que facilitará o acesso em caso de alguma dúvida referente a algum desses medicamentos. A utilização desse aplicativo é multiprofissional. No menu é possível acessar individualmente cada uma das etapas do processo, o que facilita que o profissional se dirija ao o domínio em que irá atuar naquele momento, por exemplo, o enfermeiro pode verificar os cuidados do preparo e futuramente da administração dos medicamentos. Pode também acessar as outras etapas para conhecimento de todas as barreiras necessárias ao processo.

$\mathrm{Na}$ tela de cada um dos cinco domínios foi colocado o título dos domínios, seus itens e dentro 
desses os subitens que deve sem clicados - check list-, conforme a execução da ação, assim estará checando a sua execução. No ícone (?), o profissional encontrará algumas informaçóes complementares e a justificativa daquele item.

A forma como foi construído o protótipo do aplicativo foi pautada nas contribuiçóes sugeridas pela avaliação de usabilidade, para que futuramente seja possível realizar sua validação com os profissionais que utilizaráo o protocolo. Podemos citar a avaliação heurística e dentre elas, pode-se citar as heurísticas de Nielsen. Buscou-se seguir os passos sugeridos por ele, como o menu em que profissional terá a liberdade de escolher o local que iniciará, o check list que se assemelha ao cotidiano impresso do usuário além de garantir que o formato de check list se repete em todos os domínios, favorecendo a consistência de padrôes ${ }^{(14)}$.

E ao finalizar o passo a passo do domínio quando algum cuidado não foi checado, aparecerá uma mensagem -feedback- informando que o profissional estará correndo algum risco relacionado a segurança do paciente. Foram utilizadas cores neutras, e as informaçóes de forma concisa, porém, que contemplassem as açóes para o cuidado seguro.

O feedback é também um item elementar que favorece a avaliação da usabilidade, acrescido da estética e design minimalista com títulos curtos, contribuindo para adesão ao uso do aplicativo ${ }^{(14)}$. Para melhorar os recursos da tecnologia, há também um ícone "Capacitação" que levará a uma tela que será desenvolvida futuramente com links de artigos científicos sobre o tema MPP e atividades interativas que possibilitem o profissional a atualizar-se e capacitar-se sobre o tema, proporcionando maior segurança nas suas açóes.

Um estudo que realizou uma revisão integrativa sobre aplicativos móveis em saúde no Brasil, mostrou que a categoria profissional mais beneficiada no desenvolvimento de aplicativos móveis para a área de saúde foi a multiprofissional o que contribui para um cuidado seguro, visto que todos os membros da equipe devem trabalhar em consonância e com conhecimento do cuidado como um todo sobre o paciente e não somente sobre a sua função a ser executada ${ }^{(8)}$.

Utilizar dispositivos móveis para educação e capacitação, justifica-se pelo número expressivo de usuários, quando os profissionais são orientados aos melhores momentos e forma adequada de utilização, passam a ter o controle da própria aprendizagem. $\mathrm{O}$ uso de dispositivos móveis podem ser um aliado dentro das instituições de saúde estimulando o profissional a sempre estar atualizado ${ }^{(10)}$.

Com a possibilidade do uso do aplicativo para capacitação, o profissional terá a liberdade para escolher o momento mais adequado dentro de seu cotidiano e qual conteúdo mais lhe interessa naquele momento para se estudado. Essa modalidade difere-se das comumente utilizadas com capacitaçóes presenciais e em grupo.

$\mathrm{O}$ uso de um aplicativo para dispositivos móveis para capacitação em saúde contribui para o acesso dos profissionais ao conhecimento, sem restrição de tempo e espaço, agregam informações e possibilitam maior interesse da sua atualização, visto que permitem a sua adequação ao seu ritmo de trabalho e a facilidade de acesso em diversos momentos ${ }^{(15)}$.

O ícone de referências utilizadas no conteúdo da construção desse aplicativo torna-se importante, uma vez que é necessário que se trabalhe com evidências científicas no momento da construção do conteúdo dos aplicativos. Um estudo ${ }^{(16)}$ que buscou identificar e avaliar aplicativos para dispositivos móveis relacionados à prevenção e tratamento do HIV e outras Doenças/Infecçóes Sexualmente Transmissíveis, percebeu que um dos fatores que contribui para baixa adesão é a ausência do conteúdo ser baseado em evidências científicas.

\section{CONCLUSÃO}

O protótipo de um aplicativo móvel, conforme relatado neste artigo, poderá proporcionar aos profissionais de saúde o acesso rápido a um protocolo que apresentará cuidados essenciais no momento da realização de um medicamento potencialmente perigoso.

O protótipo apresenta-se promissor para proporcionar ao profissional acesso as informações de forma instantânea e fácil enquanto utiliza um medicamento potencialmente perigoso desde a prescrição até a avaliação após a administração, ele poderá seguir os itens do protocolo passo a passo, garantindo com que as barreiras sejam executadas e ocorra a prevenção ao erro.

Poderá contribuir para a capacitação dos profissionais tanto para o conhecimento de 
quais são os MPPs e quais açóes necessárias a serem seguidas. Possibilitará que as instituiçóes conheçam por meio de evidências científicas quais as melhorias necessárias a serem realizadas para proporcionar segurança aos pacientes.

Como limitação dessa pesquisa destaca-se a necessidade da continuidade com a complementação de dados no item "Capacitação" e a inclusão dos links das bulas de cada medicamento. Após essas

\section{REFERÊNCIAS}

1. Roque KE, Tonini T, Melo ECP. Adverse events in the intensive care unit: impact on mortality and length of stay in a prospective study. Cad Saúde Pública [Internet]. 2016 [citado 2019 dec 13]; 32(10): e00081815. Disponível em: http://dx.doi. org/10.1590/0102-311X00081815

2. Carlessi KC, Padilha KG, Toffoletto MC, Henríquez-Roldán C, Juan MAC. Patient Safety Incidents and Nursing Workload. Rev Latinoam Enferm [Internet]. 2017 [citado 2019 dec 13]; 25: e2841. Disponível em: http://dx.doi. org/10.1590/1518-8345.1280.2841

3. Costa NN, Silva AEBC, Lima JC, Barbosa MRS, Freitas JS, Bezerra ALQ. The portrait adverse events in a medical clinic: analysis of a decade. Cogitare Enferm [Internet]. 2016 [citado 2019 dec 20]; 21(n. esp.): 1-10. Disponível em: http://dx.doi. org/10.5380/ce.v2li5.45661

4. Instituto para Práticas Seguras no Uso de Medicamentos potencialmente perigosos de uso hospitalar e ambulatorial - listas atualizadas [Internet]. 2015 [citado 2019 mai 14]. Disponível em: http://www.ismp-brasil.org/site/institucional/ quemsomos/

5. Rosa MB, Perini E, Anacleto TA, Neiva HM, Bogutchi T. Errors in hospital prescriptions of high-alert medications. Rev Saúde Pública [Internet]. 2009 [citado 2019 dec 20]; 43(3): 490498. Disponível em: http://dx.doi.org/10.1590/ S0034-89102009005000028

6. Agência Nacional de Saúde Suplementar (BR). O processo de elaboração, validaçâo e implementação das diretrizes clínicas na saúde suplementar no Brasil. Associação Médica Brasileira, Conselho Federal de Medicina [Internet]. Rio de Janeiro: ANS, 2009. [citado $21 \mathrm{dez}$ 2017]. Disponível em: http://bvsms.saude.gov.br/bvs/publicacoes/ processo_elaboracao_validacao_suplementar.pdf

7. Agência Nacional de Vigilância Sanitária (BR). complementações serão realizadas as validações do aplicativo e definida as plataformas que serão disponibilizadas.

A forma de preenchimento das etapas em forma de check list objetiva a agilidade no momento da utilização e propóe futuramente a possibilidade de armazenamento desses dados, o link com software do hospital e a possibilidade de gerenciar os indicadores do protocolo.

Assistência Segura: uma reflexão teórica aplicada à prática [Internet]. Série Segurança do Paciente e Qualidade em Serviços de Saúde. 2a ed. Brasília: ANVISA, 2013. 170 p. Disponível em: http:// portal.anvisa.gov.br/documents/33852/3507912/ Caderno+1+-+Assistencia+Segura+-+Uma+Reflex ao+Teorica+Aplicada+a+Pratica/97881798-cea04974-9d9b-077528ea1573

8. Tibes CMS, Dias JD, Zem-Mascarenhas SH. Mobile applications developed for the health sector in Brazil: an integrative literature review. Rev Min Enferm [Internet]. 2014 [citado 2019 dec 14]; 18(2): 479-486. Disponível em: http://dx.doi. org/10.5935/1415-2762.20140035

9. Mather CA, Gale F, Cummings EA. Governing mobile technology use for continuing professional development in the Australian nursing profession. BMC Nurs [Internet]. 2017 [citado 2019 dec 14]; 16:17. Disponível em: http://dx.doi.org/10.1186/ s12912-017-0212-8

10. Galvão ECF, Püschel VAA. Multimedia application in mobile platform for teaching the measurement of central venous pressure. Rev Esc Enferm USP [Internet]. 2012 [citado 2019 dec 20]; 46(esp): 107-115. Disponível em: http://dx.doi. org/10.1590/S0080-62342012000700016

11. Mamta. Nursing informatics: the future now. Nurs J India [Internet]. 2014 [citado 2019 dec 20]; 105(5): 198-199. Disponível em: http://www. iosrjournals.org/iosr-jnhs/papers/vol3-issue2/ Version-4/J03245153.pdf

12. Rezende LCM, Santos SR, Medeiros AL. Assessment of a prototype for the Systemization of Nursing Care on a mobile device. Rev Latinoam Enferm [Internet]. 2016 [citado 2019 dec 20]; 24: e2714. Disponível em: http://dx.doi. org/10.1590/1518-8345.0898.2714

13. Garrett JJ. The Elements of User Experience. USA: New Readers, 2a ed [Internet]. 2000 [citado 2019 mai 14]. 192 p. Disponível em: http://www.jjg.net/ elements/pdf/elements.pdf 
14. Carvalho LR, Évora YDM. Zem-Mascarenhas $\mathrm{SH}$. Assessment of the usability of a digital learning technology prototype for monitoring intracranial pressure. Rev Latinoam Enferm [Internet]. 2016 [citado 2019 dec 20]; 24(1): e2777. Disponível em: http://dx.doi.org/10.1590/1518-8345.1054.2777

15. Barra DCC, Paim SMS; Sasso GTMDal; Colla GW. Methods for developing mobile apps in health: an integrative review of the literature. Texto cont enferm [Internet]. 2017 [citado 2019 dec 20]; 26(4): e2260017. Disponível em: http:// dx.doi.org/10.1590/0104-07072017002260017

16. Muessig KE, Pike EC, Legrand S, HightowWeidman LB. Mobile phone applications for the care and prevention of HIV and other sexually transmitted diseases: a review. J Med Internet Res. 2013 [citado 2019 dec 20]; 15(1): e1. Disponível em: http://dx.doi.org/10.2196/jmir.2301 